ACCOUNTING FOR SLAVERY 



\title{
Accounting for Slavery
}

\author{
MASTERS AND MANAGEMENT
}

Caitlin Rosenthal

\author{
II \\ Harvard University Press \\ Cambridge, Massachusetts \\ London, England \\ 2018
}


Copyright $(\mathcal{C} 2018$ by the President and Fellows of Harvard College

All rights reserved

Printed in the United States of America

First printing

Library of Congress Cataloging-in-Publication Data

Names: Rosenthal, Caitlin, author.

Title: Accounting for slavery : masters and management / Caitlin Rosenthal.

Description: Cambridge, Massachusetts : Harvard University Press, 2018.

Includes bibliographical references and index.

Identifiers: LCCN 2017058060 | ISBN 9780674972094 (hardcover : alk. paper) Subjects: LCSH: Slavery—Economic aspects—United States-HistoryI8th century. | Slavery—Economic aspects-United States-HistoryIgth century. | Slavery—Economic aspects—West Indies, British—HistoryI8th century. | Slavery-Economic aspects-West Indies, British-HistoryIgth century. | Human capital—United States—History. | Human capitalWest Indies, British—History. | Plantations—United States—AccountingHistory. | Plantations-West Indies, British-Accounting-History. | Plantation owners-United States-History. | Plantation ownersWest Indies, British—History.

Classification: LCC HT905.R67 20I8 | DDC 331.II/734097309033—dc23

LC record available at https:/ / lccn.loc.gov/2017058060

Cover Design: Tim Jones

Cover Images:

Background: Ledger book from the Eli J. Capell Family Papers Collection, courtesy of Louisiana State University.

Inset: Picking cotton near Montgomery, Alabama, c r860, by J. H. Lakin, courtesy of the Library of Congress. 
For my parents, Jim and Cindy 
